\title{
Electrocardiogram Findings in Patients with Alopecia Areata
}

\author{
Danielle Peterson · Carlos Wambier · Feng Dai · Rachel Lampert • \\ Tariq Ahmad · Katerina L. Yale · Natasha A. Mesinkovska • \\ Brett King (D)
}

Received: August 11, 2021 / Accepted: September 11, 2021 / Published online: September 26, 2021

(c) The Author(s) 2021

\section{ABSTRACT}

Introduction: While autoimmune comorbidities are common in alopecia areata, little is known about comorbid cardiovascular disease. The purpose of this study was to evaluate the incidence of bradyarrhythmia in patients with alopecia areata.

Danielle Peterson and Carlos Wambier contributed equally to the work.

D. Peterson · B. King $(\bowtie)$

Department of Dermatology, Yale School of

Medicine, New Haven, CT, USA

e-mail: brett.king@yale.edu

C. Wambier

Department of Dermatology, Brown University, Providence, RI, USA

F. Dai

Center for Analytical Sciences, Yale School of

Medicine, New Haven, CT, USA

R. Lampert · T. Ahmad ( $\square)$

Department of Internal Medicine, Section of

Cardiovascular Medicine, Yale School of Medicine,

New Haven, CT, USA

e-mail: tariq.ahmed@yale.edu

K. L. Yale - N. A. Mesinkovska

Department of Dermatology, University of

California, Irvine, Irvine, CA, USA
Methods: Retrospective review of electrocardiograms of 124 patients with $\geq 50 \%$ scalp hair loss (severe alopecia areata) was conducted and compared to National Health and Nutrition Examination Survey (NHANES) data.

Results: The prevalence of bradycardia in females with alopecia areata was $24.3 \%$ (95\% CI, 14.5-34.1\%) and in those age 40 years or older was $40.8 \%$ (95\% CI, 22.2-53.5\%) compared to $19.5 \%$ in the NHANES III population. The prevalence of bradycardia in males with alopecia areata was $36.0 \%$ (95\% CI, 22.7-49.3\%) and in those age 40 years or older was $50.0 \%$ (95\% CI, $21.7-78.3 \%$ ) compared to $26.9 \%$ in the NHANES III population.

Conclusion: The potential association between bradycardia and alopecia areata merits further investigation.

Keywords: Alopecia areata; Bradycardia; Heart rate; Cardiovascular; Autoimmune; $\mathrm{T}$ cell; Electrocardiogram 


\section{Key Summary Points}

Why carry out this study?

While autoimmune comorbidities are common in alopecia areata (AA), little is known about comorbid cardiovascular disease.

The purpose of this study was to evaluate the incidence of bradycardia in patients with AA.

\section{What was learned from the study?}

Our data suggest there is increased prevalence of sinus bradycardia in AA patients.

Further investigation of the prevalence of bradycardia and its relationship to cardiovascular disease in AA patients is warranted.

\section{INTRODUCTION}

Alopecia areata (AA) is an autoimmune disease characterized by non-scarring hair loss. Comorbid cardiovascular disease in patients with autoimmune disorders is an area of active investigation. In AA patients, higher levels of cardiac troponin I have been documented [1]. One recent study suggests AA patients are at an increased risk of acute myocardial infarction [2], while another study found elevated inflammatory and cardiovascular/atherosclerosis-related biomarkers in AA patients [3]. Previous work has not suggested a connection between AA and arrhythmias. We sought to quantify the prevalence of sinus bradycardia in AA patients.

\section{METHODS}

This retrospective study involved AA patients who underwent stringent screening protocols, including electrocardiogram (ECG), for therapeutic clinical trials between October 2015 and
January 2020 at two institutions with approval from both Yale institutional review board (HIC\# 2000026876) and UCI institutional review board (HS\# 2016-3076). Screening data included patients' age, gender, severity of scalp hair loss [assessed by Severity of Alopecia Tool (SALT) score $=\%$ scalp hair loss], ECG findings, comorbidities, current medications, body mass index (BMI), and laboratory values. Bradycardia was defined as a heart rate $<60$ beats per minute (bpm). Findings were compared to ECG heart rate data from males and females in the general population (NHANES III 1988-1994) [4].

\section{Statistical Analysis}

Summary statistics were calculated as mean (standard deviation (SD)) for continuous variables and counts (\%) for categorical variables. Twosample Welch's $t$-test was used to compare mean differences of continuous variables between patients with bradycardia and patients without bradycardia, while the chi-square test was used for assessing the association of bradycardia with categorical variables. Analyses were performed using the statistical software SAS version 9.4 (Cary, NC). A two-sided $p$-value < 0.05 was considered statistically significant.

\section{RESULTS}

A total of 124 patients were included (Table 1). All patients had AA with SALT score $\geq 50$ (i.e., $50 \%$ or more scalp hair loss). Of this cohort, 74 were female $(59.7 \%)$. The mean (SD) age for all patients was 36.4 (15.4). The mean (SD) age for the NHANES III population was 57.0 (12.7), with all patients age 40 or older.

The prevalence of sinus bradycardia in our cohort was $29.0 \%$ compared to $22.9 \%$ in the NHANES III population. The prevalence of bradycardia in females with AA was $24.3 \%$ (95\% CI, $14.5-34.1 \%)$ and in those age 40 years or older was $40.8 \%$ (95\% CI, 22.2-53.5\%), compared to $19.5 \%$ in the NHANES III population. The prevalence of bradycardia in males with AA was $36.0 \%$ (95\% CI, 22.7-49.3\%) and in those age 40 years or older was $50.0 \%$ (95\% CI, $21.7-78.3 \%)$, compared to $26.9 \%$ in the 
Table 1 Summary statistics of patient variables at the screening visit

\begin{tabular}{|c|c|c|c|c|}
\hline & $\begin{array}{l}\text { ECG HR } \geq 60 \\
(n=88)\end{array}$ & $\begin{array}{l}\text { Bradycardia } \\
(\text { ECG HR < 60) } \\
(n=36)\end{array}$ & $\begin{array}{l}\text { Total } \\
(n=124)\end{array}$ & $p$ value \\
\hline Age (years) & $34.8(15.81)$ & $40.3(13.75)$ & $36.4(15.39)$ & 0.07 \\
\hline SALT score at screen & $84.6(20.01)$ & $82.4(21.70)$ & $83.9(20.45)$ & 0.58 \\
\hline Years since diagnosis & $11.4(9.53)$ & $11.4(9.75)$ & $11.4(9.56)$ & 0.98 \\
\hline Duration of current episode of severe AA (years) & $3.5(4.03)$ & $4.5(4.00)$ & $3.8(4.03)$ & 0.21 \\
\hline Systolic blood pressure & $123(12.32)$ & $123(15.15)$ & $123(13.15)$ & 0.91 \\
\hline Diastolic blood pressure & $78(10.10)$ & $79(10.71)$ & $78(10.24)$ & 0.98 \\
\hline ECG heart rate & $71(9.66)$ & $54(4.91)$ & $66(11.57)$ & $<0.001$ \\
\hline TSH $(\mu \mathrm{IU} / \mathrm{mL})$ & $1.85(0.94)$ & $1.76(1.07)$ & $1.83(0.98)$ & 0.68 \\
\hline Height $(\mathrm{cm})$ & $163.5(20.00)$ & $169.8(9.27)$ & $165.3(17.77)$ & 0.021 \\
\hline Weight $(\mathrm{kg})$ & $81.0(30.17)$ & $73.3(15.86)$ & $78.8(27.01)$ & 0.07 \\
\hline Body mass index & $26.9(5.79)$ & $25.3(4.23)$ & $26.5(5.42)$ & 0.09 \\
\hline Hemoglobin $(\mathrm{g} / \mathrm{dL})$ & $14.13(1.29)$ & $14.16(1.36)$ & $14.14(1.31)$ & 0.91 \\
\hline \multicolumn{5}{|l|}{ Sex } \\
\hline Female & $56(63.64 \%)$ & $18(50.00 \%)$ & $74(59.68 \%)$ & \multirow[t]{2}{*}{0.16} \\
\hline Male & $32(36.36 \%)$ & $18(50.00 \%)$ & $50(40.32 \%)$ & \\
\hline \multicolumn{5}{|l|}{ Hypothyroidism } \\
\hline Yes & $13(15.12 \%)$ & $6(17.14 \%)$ & $19(15.70 \%)$ & \multirow[t]{2}{*}{0.78} \\
\hline No & $73(84.88 \%)$ & $29(82.86 \%)$ & $102(84.30 \%)$ & \\
\hline \multicolumn{5}{|l|}{ Levothyroxine } \\
\hline Yes & $10(11.76 \%)$ & $5(13.89 \%)$ & $15(12.40 \%)$ & \multirow[t]{2}{*}{0.77} \\
\hline No & $75(88.24 \%)$ & $31(86.11 \%)$ & $106(87.60 \%)$ & \\
\hline \multicolumn{5}{|l|}{ Beta blocker } \\
\hline Yes & $4(4.65 \%)$ & $0(0.00 \%)$ & $4(3.28 \%)$ & \multirow[t]{2}{*}{0.32} \\
\hline No & $82(95.35 \%)$ & $36(100.00 \%)$ & $118(96.72 \%)$ & \\
\hline \multicolumn{5}{|l|}{ Calcium channel blocker } \\
\hline Yes & $2(2.33 \%)$ & $0(0.00 \%)$ & $2(1.64 \%)$ & \multirow[t]{2}{*}{1.00} \\
\hline No & $84(97.67 \%)$ & $36(100.00 \%)$ & $120(98.36 \%)$ & \\
\hline \multicolumn{5}{|l|}{ Electrolytes normal } \\
\hline Yes & $74(86.05 \%)$ & $30(83.33 \%)$ & $104(85.25 \%)$ & \multirow[t]{2}{*}{0.70} \\
\hline No & $12(13.95 \%)$ & $6(16.67 \%)$ & $18(14.75 \%)$ & \\
\hline History of cardiovascular disease ${ }^{a}$ & & & & \\
\hline
\end{tabular}


Table 1 continued

\begin{tabular}{lcccc}
\hline HR $\geq \mathbf{6 0}(\boldsymbol{n}=\mathbf{8 8})$ & ECG & & & \\
Bradycardia(ECG HR $<\mathbf{6 0})(\boldsymbol{n}=\mathbf{3 6})$ & & Total $(\boldsymbol{n}=\mathbf{1 2 4})$ & $\boldsymbol{p}$ value & Yes \\
$7(8.14 \%)$ & $2(5.56 \%)$ & $9(7.38 \%)$ & 1.00 & \\
No & $79(91.86 \%)$ & $34(94.44 \%)$ & $113(92.62 \%)$ & \\
\hline
\end{tabular}

Data are presented as mean (SD), $n(\%)$

$A A$ alopecia areata, ECG electrocardiogram, $H R$ heart rate, SALT Severity of Alopecia Tool, TSH thyroid-stimulating hormone

${ }^{a}$ Cardiovascular disease included any of the following: hypertension, prior myocardial infarction, previous diagnosis of coronary artery disease, hyperlipidemia, or cardiac conduction abnormalities

NHANES III population. In our cohort of patients with bradycardia, the mean (SD) HR was $53.6 \mathrm{bpm}$ (4.91), and there was a trend toward higher mean age in those with bradycardia (40.3 vs. 34.8 years old; $p=0.07$ ).

No association was found between bradycardia and blood pressure $(p=0.91)$, bradycardia and history of cardiovascular disease $(p=1.00)$, and bradycardia and hypothyroidism $(p=0.78)$. TSH values were similar in the two groups (1.76 vs. $1.85, p=0.68)$ (Table 1$)$. While BMI was slightly lower among bradycardic patients (25.29 vs. 26.93), this was not statistically significant $(p=0.09)$. No bradycardic patients were taking beta blockers or calcium channel blockers. Bradycardia was not associated with electrolyte abnormalities $(p=0.70)$ or anemia $(p=0.91)$.

Other ECG abnormalities were seen in 33 patients (37.5\%) without bradycardia and 11 patients (30.6\%) with bradycardia and included: early repolarization, ectopic premature complexes, incomplete right bundle branch block, intraventricular conduction delay, left axis devation, left ventricular hypertrophy, nonspecific T-wave abnormality, premature atrial contractions, right atrial enlargement, right and left ventricular hypertrophy, sinus arrhythmia, short PR interval, ST elevation, right ventricular conduction delay, and premature ventricular contractions.

\section{DISCUSSION}

Our data suggest there is an increased prevalence of sinus bradycardia in AA patients, reaching significance in female patients. This observation emerged from the relatively large number of patients with AA at our centers participating in therapeutic clinical trials. The fact that vital signs are rarely performed (and ECGs never) as part of routine dermatologic care may explain why this potentially novel finding has escaped notice.

Whereas sinus bradycardia suggests cardiovascular health in the general population, the clinical implications of this finding in AA patients is unknown and the mechanism underlying this association unclear. The lifetime prevalence of AA is $2 \%$ in the US population, and autoimmune and inflammatory comorbid diseases are common in patients with AA. Thyroid disorders including subclinical hypothyroidism, Graves' disease, and Hashimoto's are particularly common. While hypothyroidism may cause bradycardia, there was no association between a history of hypothyroidism and the presence of bradycardia in our cohort; furthermore, all patients in our cohort who had a diagnosis of hypothyroidism were adequately treated with thyroid replacement therapy and had normal TSH values at the time ECGs were performed. A common cause of slower HR is physical 
conditioning. While activity patterns of the AA cohort were not queried, BMI did not significantly differ between those with and without bradycardia, making differences in physical conditioning an unlikely explanation. Furthermore, in our experience AA patients do not differ significantly from the general population in terms of lifestyle, further arguing that fitness is unlikely to account for the differences between the AA cohort and the NHANES population.

There are a few possible mechanisms by which sinus bradycardia could manifest in AA. Heart rate is determined by two factors-intrinsic sinus node function and autonomic activity. Inflammatory processes would be expected to increase sympathetic activity and decrease vagal activity, leading to increases in heart rate through autonomic influence on the sinus node; thus, autonomic influences would not explain our finding. Ischemia can lead to bradycardia, but we noted no ECG findings or symptoms suggestive of coronary artery disease in our patients.

Another explanation for sinus bradycardia in patients with AA could be fibrosis. Primary sinus node dysfunction is often caused by fibrosis, and, interestingly, cardiac remodeling and fibrosis have been found in murine models of AA, although conduction system or sinus node disease has not specifically been reported in these models [5, 6]. Higher levels of cardiac troponin I have been documented in patients with AA [1], and, in a murine model, an autoimmune response to cardiac troponin I induces cardiac inflammation and fibrosis $[7,8]$. It is also possible that genes involved in development of sinus node fibrosis overlap with those that determine AA $[9,10]$.

\section{Study Limitations}

Our data were limited by a relatively small cohort of AA patients in a clinical trial setting with more females (59.7\%), and the mean age of our cohort was 20 years younger than NHANES III, which collected ECG data only in patients age 40 and older. To our knowledge, there are no publicly available ECG data in populations of similar age to our AA cohort. Lastly, cardiac magnetic resonance imaging (MRI) was not performed as part of this study.

\section{CONCLUSION}

In summary, our data suggest there is increased prevalence of sinus bradycardia in AA patients. Further investigation of the prevalence and mechanism of bradycardia in AA patients is warranted.

\section{ACKNOWLEDGEMENTS}

Funding. This work was supported by the Ranjini and Ajay Poddar Fund for Dermatologic Diseases Research (Brett King). The Rapid Service Fee was funded by the authors.

Authorship. All named authors meet the International Committee of Medical Journal Editors (ICMJE) criteria for authorship for this article, take responsibility for the integrity of the work as a whole, and have given their approval for this version to be published.

Authors' Contributions. All authors contributed to the study conception and design. Material preparation, data collection, and analysis were performed by Danielle Peterson, Carlos Wambier, Katerina Yale, Natasha Mesinkovska, and Brett King. The first draft of the manuscript was written by Danielle Peterson and all authors commented on previous versions of the manuscript. All authors read and approved the final manuscript.

Prior Presentation. This work was presented at the Yale Postdoctoral Association Symposium in New Haven, CT, on 17 June 2021.

Disclosures. Brett King and Natasha Mesinkovska are investigators for Concert Pharmaceuticals Inc, Eli Lilly and Company, and Pfizer Inc. Brett King is a consultant to and/or has served on advisory boards for Aclaris Therapeutics, Arena Pharmaceuticals, Bristol-Meyers 
Squibb, Concert Pharmaceuticals Inc., Dermavant Sciences, Eli Lilly and Company, Pfizer Inc., and Viela Bio; he is on speaker's bureau for Regeneron and Sanofi Genzyme. Natasha Mesinkovska is a consultant for the National Alopecia Areata Foundation, Arena Pharmaceuticals, and Concert Pharmaceuticals Inc. Carlos Wambier is an investigator for Concert and Pfizer. Danielle Peterson, Feng Dai, Rachel Lampert, Tariq Ahmad, and Katerina Yale have nothing to disclose.

Compliance with Ethics Guidelines. Reviewed and approved by Yale IRB, HIC \# 2000026876, and UCI IRB, HS\#2016-3076. Study was performed in accordance with the Helsinki Declaration of 1964 and its later amendments. All subjects provided informed consent to participate in clinical trials at their respective center.

Data Availability. Data sharing is not applicable to this article as no datasets were generated or analyzed during the current study.

Open Access. This article is licensed under a Creative Commons Attribution-NonCommercial 4.0 International License, which permits any non-commercial use, sharing, adaptation, distribution and reproduction in any medium or format, as long as you give appropriate credit to the original author(s) and the source, provide a link to the Creative Commons licence, and indicate if changes were made. The images or other third party material in this article are included in the article's Creative Commons licence, unless indicated otherwise in a credit line to the material. If material is not included in the article's Creative Commons licence and your intended use is not permitted by statutory regulation or exceeds the permitted use, you will need to obtain permission directly from the copyright holder. To view a copy of this licence, visit http://creativecommons.org/licenses/by$\mathrm{nc} / 4.0 /$.

\section{REFERENCES}

1. Wang E, Santos L, Li X, Tran A, Kim S, Woo K, et al. Alopecia areata is associated with increased expression of heart disease biomarker cardiac troponin I. Acta Derm Venereol. 2018;98(8):776-82. https://doi.org/10.2340/00015555-2964.

2. Shin J-W, Kang T, Lee JS, Kang MJ, Huh C-H, Kim $\mathrm{M}-\mathrm{S}$, et al. Time-dependent risk of acute myocardial infarction in patients with alopecia areata in Korea. JAMA Dermatol. 2020;156(7):763-71. https://doi. org/10.1001/jamadermatol.2020.1133

3. Glickman JW, Dubin C, Renert-Yuval Y, Dahabreh D, Kimmel GW, Auyeung K, et al. Cross-sectional study of blood biomarkers of patients with moderate-to-severe alopecia areata reveals systemic immune and cardiovascular biomarker dysregulation. J Am Acad Dermatol. 2020;84(2):370-80. https://doi.org/10.1016/j.jaad.2020.04.138

4. Centers for Disease Control and Prevention (CDC). National Center for Health Statistics (NCHS). National Health and Nutrition Examination Survey Questionnaire (or Examination Protocol, or Laboratory Protocol). Hyattsville, MD: U.S. Department of Health and Human Services, Centers for Disease Control and Prevention, 1988-1994. https://wwwn. cdc.gov/nchs/nhanes/nhanes3/default.aspx. Accessed 17 Mar 2020

5. Wang E, Chong $\mathrm{K}, \mathrm{Yu} \mathrm{M}$, Akhoundsadegh $\mathrm{N}$, Granville DJ, Shapiro J, et al. Development of autoimmune hair loss disease alopecia areata is associated with cardiac dysfunction in $\mathrm{C} 3 \mathrm{H} / \mathrm{HeJ}$ mice. Beeton C, editor. PLoS ONE. 2013;8(4): e62935. https://doi.org/10.1371/journal.pone. 0062935

6. Sundberg JP, Chevallier L, Silva KA, Kennedy VE, Sundberg BA, Li Q, et al. Mouse alopecia areata and heart disease: know your mouse!. J Invest Dermatol. 2014;134(1):279-81. https://doi.org/10.1038/jid. 2013.273.

7. Göser S, Andrassy M, Buss SJ, Leuschner F, Volz CH, Öttl R, et al. Cardiac troponin I but not cardiac troponin $\mathrm{T}$ induces severe autoimmune inflammation in the myocardium. Circulation. 2006;114(16): 1693-702. https://doi.org/10.1161/ CIRCULATIONAHA.106.635664

8. Kaya Z, Göser S, Buss SJ, Leuschner F, Öttl R, Li J, et al. Identification of cardiac troponin I sequence motifs leading to heart failure by induction of myocardial inflammation and fibrosis. Circulation. 2008;118(20):2063-72. https://doi.org/10.1161/ CIRCULATIONAHA.108.788711. 
9. Peng H, Sarwar Z, Yang X-P, Peterson EL, Xu J, Janic $B$, et al. Profibrotic role for interleukin-4 in cardiac remodeling and dysfunction. Hypertension. 2015;66(3):582-9. https://doi.org/10.1161/ HYPERTENSIONAHA.115.05627.
10. Petukhova L, Duvic M, Hordinsky M, Norris D, Price $\mathrm{V}$, Shimomura $\mathrm{Y}$, et al. Genome-wide association study in alopecia areata implicates both innate and adaptive immunity. Nature. 2010;466(7302):113-7. https://doi.org/10.1038/nature09114. 